\title{
PASAT and Components of WAIS-R Performance: Convergent and Discriminant Validity
}

\author{
J. R. Crawford \\ University of Aberdeen, $U K$ \\ M. C. Obonsawin \\ University of Strathclyde, Glasgow, UK \\ K. M. Allan \\ St. Johns Hospital, Livingston, UK
}

The Paced Auditory Serial Addition Task (PASAT) (Gronwall, 1977), a fulllength Wechsler Adult Intelligence Scale-Revised (WAIS-R) (Wechsler, 1981), and the National Adult Reading Test (NART) (Nelson, 1982) were completed by a sample of 152 healthy participants. Principal components analysis revealed that PASAT's loading on general intelligence $(g)$ was substantial (.72) and exceeded that of many WAIS-R subtests. Following rotation, PASAT loaded highly (.75) on the WAIS-R attention/concentration factor. Evaluation of nested confirmatory factor analytic models confirmed that, although PASAT is not independent of general intellectual ability, it still loaded significantly on attention/concentration (.44) when it was permitted to load simultaneously on $g$. Conventional normative data were provided for three age bands. In addition, regression equations were built to permit a quantitative comparison of individuals' obtained PASAT scores with their estimated premorbid PASAT scores (using NART and age as predictors) and with scores predicted from their current general level of intellectual ability (using WAIS-R IQ and age as predictors). Both these sets of predictor variables accounted for highly significant proportions of PASAT variance; Multiple $R$ was .52 for the former and .66 for the latter.

Slowed information processing and deficits in sustained attention are among the most common sequelae of closed head injury (CHI) (van Zomeren \& Brouwer, 1992). The Paced Auditory Serial Addition Task (PASAT)

Requests for reprints should be sent to J. R. Crawford, Department of Psychology, King's College, University of Aberdeen, Old Aberdeen, Aberdeen AB24 2UB. Email: crawford@ psyc.abdn.ac.uk. 
(Gronwall, 1977; Gronwall \& Sampson, 1974) has been widely endorsed as a means of measuring these problems (e.g. Levin, Benton, \& Grossman, 1982; McKinley \& Gray, 1992; van Zomeren \& Brouwer, 1992). PASAT requires participants to add pairs of numbers under time pressure, the second number in the pair becomes the first number of the subsequent pair thereby requiring a constant updating of working memory. There are four trials of 61 digits in the original version with digit presentation rates of 2.4, 2.0, 1.6, and 1.2 seconds.

The first aim of the present study was to provide additional normative data for the PASAT. Although adequate normative data is available for the Galveston modification of the PASAT (Brittain et al., 1991; Levin et al., 1987; Roman, Edwall, Buchanan, \& Patton, 1991) the only traceable norms for the original version are based on a sample of 80 New Zealanders consisting predominantly of young males for whom limited demographic data were available (Gronwall \& Wrightson, 1974).

A second aim was to examine whether age was related to PASAT performance. There is a consistent body of evidence demonstrating that measures of attention and speed of information processing decline with age (e.g. Salthouse, 1991). Although there is a linear component to this decline it is common for there to be an additional non-linear component (i.e. an acceleration of the apparent rate of decline in the oldest age groups). In the case of PASAT, matters are complicated by a recent report that an elderly sample outperformed a young adult sample. Ward (1997) suggested that this result may have arisen because the current generation of young adults is not as familiar with number bonds as previous generations. Given the consistency of the age effects for speeded measures he also notes that this reversal of the normal trend casts doubt on the construct validity of the PASAT. Thus, there are many potential forms for the relationship between age and PASAT performance. For example, it may be that there is a linear component associated with overall decline coupled with non-linear components at both extremes of the adult age range. In the present study the relationship between age and PASAT performance will be modelled with multiple regression in which the possibility of non-linear components will be allowed for by entering polynomial functions of age as potential predictors.

The third aim was to obtain data on the convergent and discriminant validity of PASAT by examining its relationship with the Wechsler Adult Intelligence Scale-Revised (WAIS-R) (Wechsler, 1981). Specifically, the suggestion made by the developers of PASAT that performance "is not significantly correlated with either general intelligence or arithmetic ability" (Gronwall \& Wrightson, 1981, p. 889) will be evaluated. Empirical studies of this issue have produced inconsistent results. For example, Egan (1988) reported highly significant correlations between PASAT and a number of measures of intellectual functioning in a sample of 28 healthy adolescents. Deary, Langan, Hepburn, and Frier (1991) examined the relationship between PASAT and WAIS-R 
performance using exploratory factor analysis in a sample of diabetic patients $(N=94)$ and reported that PASAT loaded highly on the first principal component (i.e. general intelligence or $g$ ). Roman et al. (1991) have also examined the relationship between PASAT and the WAIS-R in a sample of 143 healthy participants. In contrast to these previous studies, Roman et al. (1991) concluded that, "for practical purposes IQ appears to bear a minimal relationship to PASAT performance" (p. 38).

Factor analytic studies of the WAIS-R have consistently extracted a large general factor accounting for more than $50 \%$ of the subtest variance. The factor structure obtained following rotation of this factor and two additional factors consists of a verbal factor indexed by Information, Vocabulary, Comprehension, and Similarities; a perceptual organisation factor indexed primarily by Block Design and Object Assembly, and an attention/concentration, or freedom-from-distractibility factor indexed primarily by Arithmetic and Digit Span (e.g. Atkinson, Cyr, Doxey, \& Vigna, 1989; Crawford et al., 1989; Leckliter, Matarazzo, \& Silverstein, 1986). Digit Symbol is often found to have a modest loading on this third factor, thus all indicators require symbol manipulation under conditions of sustained attention.

Exploratory and confirmatory factor analyses were conducted in the present study to examine PASAT's relationship with these components of the WAIS-R. The majority of cognitive ability measures load on a factor of general intelligence. We therefore hypothesised that, contrary to Gronwall \& Wrightson's (1981) suggestion, PASAT will load on general intelligence (i.e. it will show modest discriminant validity at best) but nevertheless will possess high convergent validity as a measure of attention, as indicated by a high loading on the WAIS-R attention/concentration factor. This hypothesis was formally evaluated by testing whether a confirmatory factor analytic model, in which PASAT loaded on general intelligence and attention/concentration factors, had significantly better fit than models which did not permit such loadings.

Finally, were it to be confirmed that PASAT exhibits a strong relationship with intelligence, then performance should be interpreted relative to an estimate of premorbid intellectual ability in clinical practice (e.g. an average level of performance may represent significant impairment for an individual of high premorbid ability). To permit such a comparison it was intended to employ the present healthy sample to build a regression equation for prediction of PASAT scores from the National Adult Reading Test (NART) (Nelson, 1982; Nelson \& Willison, 1992). The NART is highly correlated with WAIS and WAIS-R IQ yet is relatively resistant to the effects of closed head injury (Crawford, Besson, \& Parker, 1988) and other neurological conditions (see Crawford, 1992; O'Carroll, 1995 for reviews). Therefore, it has the potential to provide an estimate of premorbid PASAT performance against which to compare a client's obtained score. 
It would also be useful to compare clients' PASAT scores with their general level of current intellectual functioning to determine if attentional functioning is differentially impaired. Therefore it was also intended to build a regression equation to predict PASAT performance from WAIS-R IQ scores.

\section{METHOD}

\section{Participants}

The sample consisted of 152 participants, ( 77 males, 75 females) screened by interview to exclude individuals with neurological, psychiatric, or major systemic disorders. Participants were recruited from a variety of sources including recreational clubs, community centres, the public service, and commercial organisations. Participants received a small honorarium for their participation. The mean age of the sample was 40.21 years $(\mathrm{SD}=13.89)$ with a range of $16-74$ years, and mean years of full-time education or equivalent was 12.97 ( $\mathrm{SD}=$ 2.86).

Each participant's social class was coded on the basis of their current occupation (or last occupation where a participant was not in employment) using the Office of Population Censuses and Surveys' (1980) Classification of Occupations. A Chi-square goodness-of-fit test was used to compare the distribution of social class in the present sample with the census-derived distribution of social class for the adult UK population. This revealed that the two distributions did not differ significantly $\left[\chi^{2}(4, n=152)=7.89, P>.05\right]$.

\section{Tests and Procedure}

All participants completed the NART and a full-length WAIS-R according to standard procedures (Wechsler, 1981; Nelson, 1982). The NART is an oral word reading test consisting of 50 words which are irregular in their pronunciation (e.g. chord). By convention, performance is expressed as the number of errors of pronunciation, therefore high scores represent poor performance.

The original Gronwall and Wrights on (1974) version of PASAT was administered and scored strictly according to these authors' directions. Following the standard description of the task and presentation of practice items, four trials of 61 digits were presented on a tape recorder with verified digit presentation rates of 2.4, 2.0, 1.6, and 1.2 seconds. For each participant the number of digit pairs added correctly on each trial were summed, thus the maximum score obtainable was 240 . 


\section{Statistical Analysis}

Principal components analysis with a priori extraction of three factors and varimax rotation was performed on the WAIS-R subtest scaled scores and PASAT scores.

Confirmatory (maximum likelihood) factor analysis was performed on the variance-covariance matrix of the WAIS-R subtests and PASAT scores using EQS for Windows Version 4 (Bentler, 1989; Bentler \& Wu, 1993). It was considered desirable to construct an initial model which simultaneously identified a general factor and the three specific WAIS-R factors. The method of parameterisation recommended by Gustafson and Balke (1993) was therefore employed. In this parameterisation all manifest variables (i.e. the WAIS-R subtests and PASAT) were indicators of a general factor and the WAIS-R subtests also identified their respective specific factors. The specific factors were constrained to be orthogonal to (i.e. uncorrelated with) the general factor and each other. In the present study some of the variants on this basic model had only two indicators (Digit Span and Arithmetic) of the attention/concentration factor; therefore, to avoid model underidentification, the loadings of these two indicators on this factor were constrained to be equal.

In the initial model, PASAT was permitted to load on the general and attention/concentration factors. A graphical representation of this model is presented in Fig. 1. This model was compared with three nested variants; a model is considered to be nested within another model if it differs only in imposing additional constraints on the relationships between variables specified in the initial model. In the first of these variants PASAT was constrained to have zero loadings on all factors: in the second, PASAT was constrained to load only on the attention/concentration factor, and in the final variant PASAT was constrained to load only on the general factor.

The fit of these models was assessed using Chi-square, the average offdiagonal standardised residual, and the Comparative Fit Index (CFI) (Bentler, 1989). Off-diagonal standardised residuals reflect the extent to which covariance between manifest variables has not been accounted for by the models under consideration. Values for the CFI can range from zero to unity: the CFI essentially expresses the fit of a model relative to what is termed the null model (the null model posits no relationship between any of the manifest variables). There is general agreement that a model with a CFI of less than .9 should not be viewed as providing a satisfactory fit to the data (Bentler, 1989).

The difference between Chi-square for nested models is itself distributed as Chi-square with $k$ degrees of freedom where $k$ equals the degrees of freedom for the more constrained model minus the degrees of freedom for the less constrained model. Because of this it was possible to test directly whether the more constrained models had significantly poorer fit than the initial model; this 


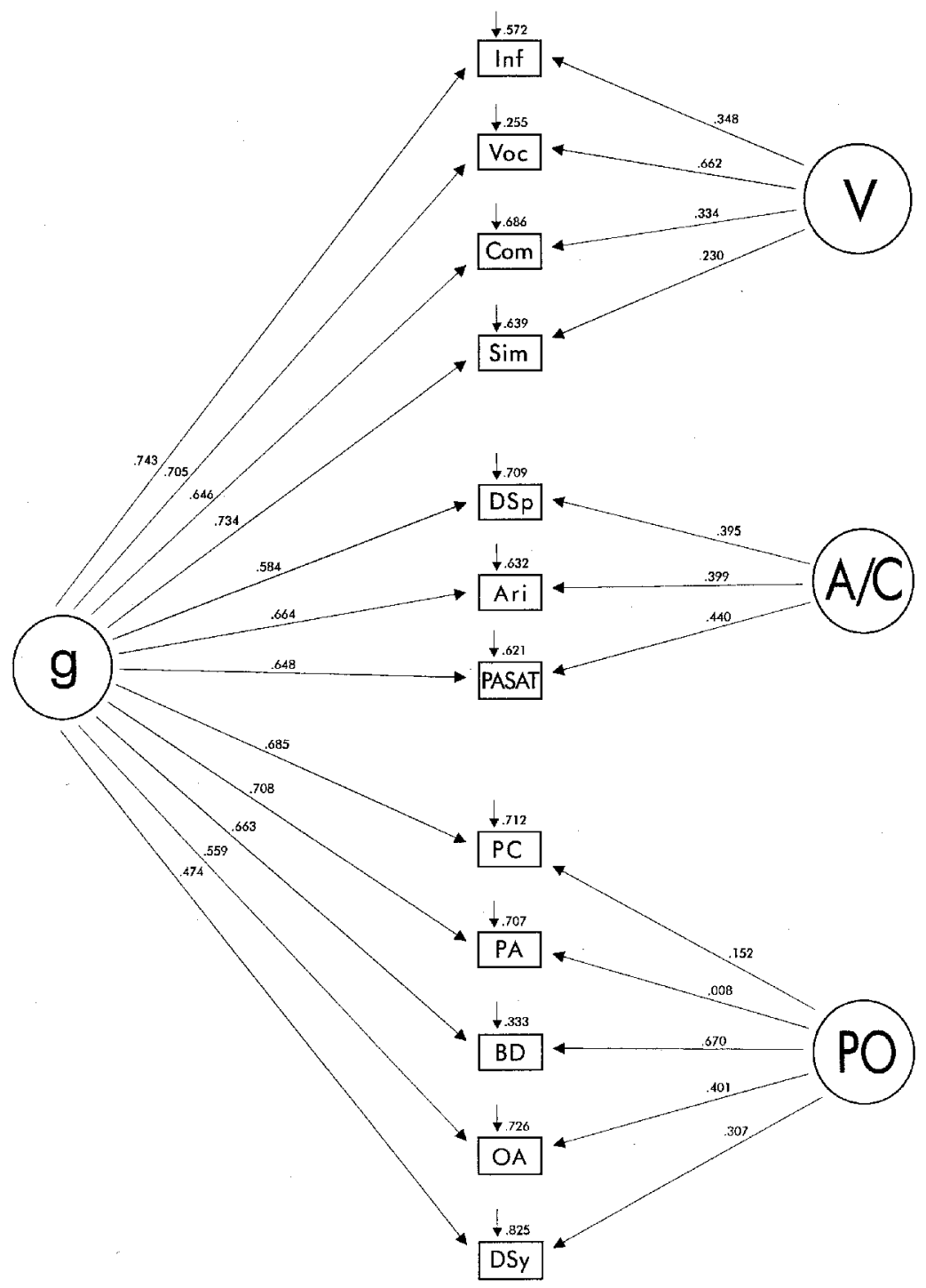

FIG. 1. Graphical representation of a confirmatory factor analytic model in which PASAT is an indicator of general intelligence and a specific attentional factor. The latent variables are represented as circles; $g=$ general, $\mathrm{V}=$ verbal, $\mathrm{A} / \mathrm{C}=$ attention/concentration, $\mathrm{PO}=$ perceptual organisation. The manifest variables are represented as squares; Inf $=$ information, Voc $=$ Vocabulary, Com $=$ Comprehension, $\operatorname{Sim}=$ Similarities, DSp $=$ Digit Span, Ari $=$ Arithmetic, $\mathrm{PC}=$ Picture Completion, PA = Picture Arrangement, BD = Block Design, OA = Object Assembly, DSy $=$ Digit Symbol, PASAT $=$ Paced Auditory Serial Addition Task. The numbers above each arrow pointing from the latent variables to manifest variables represent the standardised loadings of each manifest variable on its respective latent variables. Each manifest variable also has an error component; these are presented alongside the arrows which appear above each manifest variable. 
feature of confirmatory factor analysis (CFA) is one of its major advantages over exploratory factor analysis (EFA).

\section{RESULTS}

Summary statistics for PASAT scores, WAIS-R Full Scale IQ (FSIQ), and NART errors are presented in Table 1 . Regression analysis with PASAT as the dependent variable and age as the predictor revealed a significant but modest linear decline in PASAT performance with age $(r=.34 P<.0001)$. Addition of polynomial functions of age $\left(\mathrm{age}^{2}\right.$ and $\left.\mathrm{age}^{3}\right)$ to the regression model did not produce a significant increase in the variance predicted; thus there was no evidence for a non-linear component in the relationship in the present sample. A scatterplot of age and PASAT performance is presented as Fig. 2.

The sample was divided into three age bands (16-29, 30-49, and 50-74 years) to form a normative table. Means and standard deviations for PASAT scores in these three age bands are presented in Table 2 . The reliability (internal consistency) of PASAT was estimated by computing Cronbach's alpha from scores on the four PASAT trials. This yielded an alpha of .90 which was used to derive standard errors of measurement for each age band using the normal formula (e.g. Anastasi, 1990).

The correlation between PASAT and the Arithmetic subtest of the WAIS-R was computed to examine Gronwall and Wrightson's (1981) suggestion that PASAT is unrelated to arithmetic ability. The correlation was highly significant $(r=.63, P=<.001)$.

TABLE 1

Summary Statistics for PASAT, Full

Scale IQ (FSIQ) and NART errors

\begin{tabular}{lrr}
\hline Measure & Mean & SD \\
\hline PASAT & 151.6 & 40.32 \\
WAIS-R FSIQ & 105.0 & 14.08 \\
NART errors & 18.0 & 9.01 \\
\hline
\end{tabular}

TABLE 2

Normative Data for Gronwall and Sampson's (1974) Version of PASAT for Three Age Bands

Age Bands

\begin{tabular}{lccc}
\cline { 2 - 4 } & 16-29 years & 30-49 years & 50-74 years \\
\hline$n$ & 38 & 78 & 36 \\
Mean Age (SD) & $25.0(3.27)$ & $38.1(5.67)$ & $60.7(7.41)$ \\
Mean PASAT (SD) & $169.2(30.12)$ & $149.8(40.29)$ & $136.9(43.79)$ \\
PASAT SEM & 9.38 & 12.54 & 13.63 \\
\hline
\end{tabular}




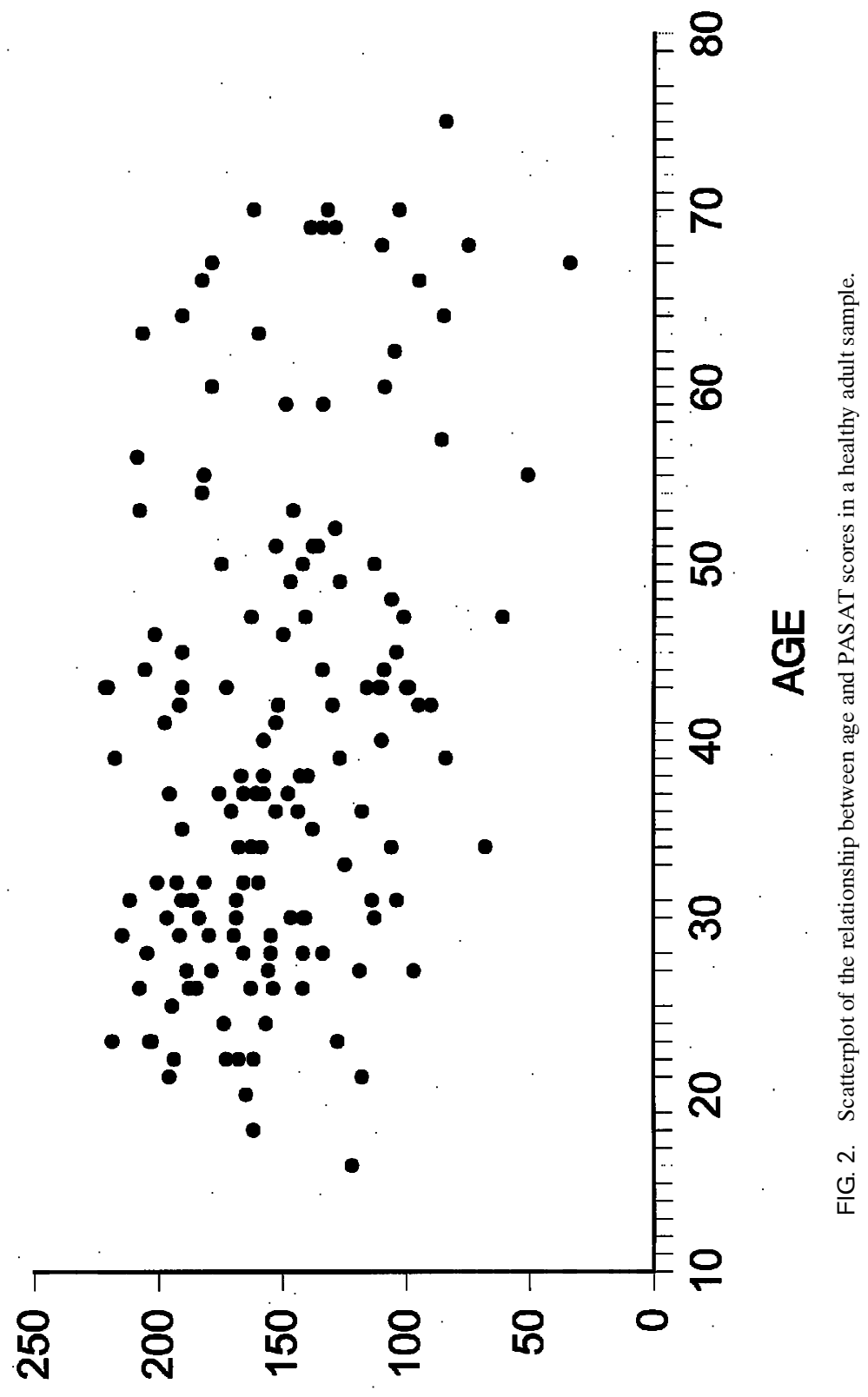

$\perp \forall S \forall d$ 
The results from the principal components analysis are presented in Table 3. The first column of this table records the loadings of the PASAT and WAIS-R subtests on the first unrotated principal component (i.e. $g$ or general intelligence). It can be seen that the PASAT loads highly on $g$ (.72). Loadings on the three factors obtained after varimax rotation are also presented in Table 3 . This factor structure is very similar to that obtained in other exploratory factor analytic studies of the WAIS-R; the present factors correspond closely to the verbal, perceptual organisation, and attention/concentration factors described previously. It can be seen from Table 3 that the PASAT loads highly (.75) on the attention/concentration factor, its loading being comparable to those of Arithmetic and Digit Span.

Confirmatory factor analyses were run on the WAIS-R/PASAT covariance matrix. As a covariance matrix is not very informative, the correlation matrix is presented in Table 4 accompanied by the standard deviations of the variables. Fit indices for the four confirmatory factor analytic models specified in the Method section are presented in Table 5. The initial model (Model 1 in Table 5) which permitted PASAT to load on the general intelligence and attention/concentration factors had a reasonable level of practical fit. Although Chi-square for this model was statistically significant, this is common in attempting to fit models of cognitive ability (Gustafson \& Balke, 1993) and, specifically, models involving the WAIS-R (e.g. O'Grady, 1983). This model meets Loehlin's (1987) criterion for a satisfactory practical level of fit which

TABLE 3

Principal Components Analysis of PASAT and the WAIS-R; Loadings on the First Unrotated Principal Component (FUPC) and on Factors Obtained Following Varimax Rotation

\begin{tabular}{|c|c|c|c|c|}
\hline & \multirow[b]{2}{*}{ FUPC } & \multicolumn{3}{|c|}{ Rotated Factors } \\
\hline & & Verbal & $P O$ & $A C$ \\
\hline Information & .79 & .76 & .18 & .35 \\
\hline Digit Span & .66 & .36 & .11 & .73 \\
\hline Vocabulary & .78 & .84 & .14 & .27 \\
\hline Arithmetic & .72 & .38 & .19 & .72 \\
\hline Comprehension & .71 & .78 & .10 & .24 \\
\hline Similarities & .78 & .72 & .35 & .19 \\
\hline Picture Completion & .72 & .56 & .58 & .06 \\
\hline Picture Arrangement & .71 & .53 & .43 & .22 \\
\hline Block Design & .73 & .26 & .78 & .28 \\
\hline Object Assembly & .63 & .27 & .77 & .06 \\
\hline Digit Symbol & .54 & .01 & .68 & .36 \\
\hline PASAT & .72 & .17 & .44 & .75 \\
\hline
\end{tabular}

$P O=$ perceptual organisation; $A C=$ attention/concentration. 
TABLE 4

Intercorrelations between Manifest Variables and Standard Deviations

\begin{tabular}{lllllllllllll}
\hline & Inf $^{\text {a }}$ & Dsp & Voc & Ari & Com & Sim & PC & PA & BD & OA & DSy & PASAT \\
\hline DSp & .475 & & & & & & & & & & & \\
Voc & .755 & .494 & & & & & & & & & & \\
Ari & .584 & .545 & .492 & & & & & & & & & \\
Com & .602 & .410 & .674 & .485 & & & & & & & & \\
Sim & .595 & .507 & .670 & .410 & .586 & & & & & & & \\
PC & .527 & .329 & .516 & .426 & .477 & .509 & & & & & & \\
PA & .514 & .398 & .501 & .404 & .451 & .570 & .489 & & & & & \\
BD & .501 & .366 & .445 & .486 & .305 & .465 & .550 & .461 & & & & \\
OA & .346 & .303 & .326 & .368 & .321 & .451 & .51 & .392 & .639 & & & \\
DSy & .252 & .316 & .241 & .264 & .256 & .362 & .362 & .369 & .525 & .355 & & \\
PASAT & .462 & .524 & .412 & .628 & .387 & .407 & .414 & .461 & .545 & .408 & .509 & \\
& & & & & & & & & & & & \\
SDs & 2.85 & 2.88 & 2.76 & 2.90 & 2.71 & 2.89 & 2.69 & 3.24 & 2.93 & 2.85 & 2.98 & 40.32 \\
\hline
\end{tabular}

${ }^{\mathrm{a}}$ See legend to Fig. 1 for key to abbreviations.

states that Chi-square should not exceed twice the degrees of freedom. Other fit indices also suggest a food fit; the CFI is high (.96) and the average off-diagonal standardised residual is low. A graphical representation of this model is presented as Fig. 1.

Fit indices for the nested variants on this model which imposed additional constraints on PASAT's loadings are also presented in Table 5. Model 2, which constrained PASAT to have zero loadings on all WAIS-R factors, led to a substantial deterioration in fit. It can be seen that the CFI for this model is low; additionally Chi-square is large and substantially exceeds twice the model's degrees of freedom. Table 6 compares the fit of the initial model with the three nested variants using the Chi-square difference test outlined earlier. It can be seen from Table 6 that Model 2 had significantly poorer fit than the initial model $(P<.00001)$.

TABLE 5

Fit Indices for WAIS-R/PASAT Confirmatory Factor Analytic Models

\begin{tabular}{lcccc}
\hline \multicolumn{1}{c}{ Model } & Chi-square & df & CFI & AODSR \\
\hline $\begin{array}{l}\text { 1. } \quad \text { + three specific factors; PASAT permitted to } \\
\text { load on } g \text { and attention/concentration }\end{array}$ & 85.04 & 43 & .96 & .038 \\
2. PASAT constrained to have zero loadings in all & 181.54 & 45 & .85 & .102 \\
$\quad \begin{array}{l}\text { WAIS-R factors } \\
\text { 3. PASAT constrained to load only on attention/ }\end{array}$ & 144.06 & 44 & .89 & .127 \\
$\begin{array}{l}\text { concentration } \\
\text { 4. PASAT constrained to load only on } g\end{array}$ & 99.78 & 44 & .94 & .045 \\
\hline
\end{tabular}

${ }^{\mathrm{a}} \mathrm{CFI}=$ Comparative Fit Index, ${ }^{\mathrm{b}}$ AODSR $=$ Average off-diagonal standardised residual. 
TABLE 6

Comparison of Model Fit for Nested CFA Models

\begin{tabular}{lccc}
\hline Comparison & Chi-square $^{\mathrm{a}}$ & $\mathrm{df}^{b}$ & \multicolumn{1}{c}{$\mathrm{P}$} \\
\hline Model 1 vs Model 2 & 96.50 & 2 & $<.0001$ \\
Model 1 vs Model 3 & 59.02 & 1 & $<.00001$ \\
Model 1 vs Model 4 & 14.73 & 1 & $<.001$ \\
\hline
\end{tabular}

${ }^{a}$ Value for Chi-square is derived by subtracting the Chi-square value for the less constrained model from the Chi-square value for the more constrained model. ${ }^{\mathrm{b}} d f=d f$ for the more constrained model minus $d f$ for the less constrained model.

Model 3 constrained PASAT to load only on the attention/concentration factor. As can be seen from Table 5 this model also had poor fit by all criteria and had significantly poorer fit $(P<.0001)$ than the initial model (see Table 6$)$. Model 4 constrained PASAT to load only on the general intelligence factor. On the basis of the CFI (.94) and average off-diagonal standardised residual this model has a reasonable fit; however, it fails to meet Loehlin's (1987) criterion. Additionally, this model had significantly poorer fit $(P<.001)$ than the initial model in which PASAT was permitted to load on the attention/concentration factor in addition to general intelligence (see Table 6).

The foregoing analyses indicate that PASAT is not independent of general intelligence. Therefore age and NART errors were used as predictors in a regression analysis to provide a means of estimating premorbid PASAT performance for use in clinical practice. A stepwise procedure was used and revealed that NART was the best predictor, accounting for a highly significant proportion of the variance in PASAT scores $(P<.001)$. Addition of age to the model produced a significant increase in $R^{2}(P<.001)$. The regression equation, Multiple $R$ and the standard error of estimate (SEest) derived from this analysis are presented in Table 7 . The SEest was multiplied by the appropriate values of $z$ to estimate the discrepancy between predicted and obtained PASAT scores required to exceed 85,90 , and $95 \%$ of the present sample. These discrepancies are presented in Table 7; note that they are "one-tailed" as a clinical practice the concern will be with whether a client's obtained PASAT score is lower than their estimated premorbid level of performance.

A stepwise regression analysis was also conducted to permit a comparison of PASAT performance against a client's current general level of intellectual functioning using WAIS-R FSIQ and age as predictors. WAIS-R was the best predictor accounting for a significant proportion of PASAT variance $(P<$ $.001)$; addition of age produced a significant $(P<.001)$ increase in $R^{2}$. The regression equation and other information equivalent to that reported earlier for the NART/PASAT equation are presented in Table 7. 


\section{TABLE 7}

Results from Regression Analyses Predicting PASAT Scores from NART Plus Age and WAIS-R FSIQ Plus Age

\begin{tabular}{|c|c|c|c|c|c|c|}
\hline \multirow[b]{2}{*}{ Predictors } & \multirow[b]{2}{*}{ Regression equations } & \multicolumn{2}{|l|}{ Multiple } & \multicolumn{3}{|c|}{$\begin{array}{l}\text { Discrepancy } \\
\text { required to exceed } \\
\text { given percentage of } \\
\text { healthy sample }\end{array}$} \\
\hline & & $R$ & SEest & $85 \%$ & $90 \%$ & $95 \%$ \\
\hline NART errors, Age & $215.74-(1.85 \times \mathrm{NART})-(.77 \times$ Age $)$ & .52 & 34.87 & 35.9 & 44.6 & 57.2 \\
\hline WAIS-R FSIQ, Age & $12.87+(1.65 \times$ FSIQ $)-(.87 \times$ Age $)$ & .66 & 30.66 & 31.6 & 39.3 & 50.3 \\
\hline
\end{tabular}

${ }^{\mathrm{a}}$ The values are one-tailed.

\section{DISCUSSION}

The major aim of the present study was to evaluate the convergent and discriminant validity of the PASAT through examining its relationship with WAIS-R performance. The results from principal components analysis of PASAT and the 11 WAIS-R subtests suggests that PASAT has reasonable convergent validity as a measure of attention/concentration. PASAT loaded highly (.75) on the attention/concentration factor identified in previous factor analytic studies of the WAIS-R. PASAT's loading was essentially equivalent to the two WAIS-R subtests which identify this factor. However, PASAT's loading on the first unrotated principal component was also high (.72) which suggests that its discriminant validity is modest. This conclusion is underlined by the magnitude of the loadings on the general factor; PASAT's loading was exceeded by only four of the 11 WAIS-R subtests.

The results of the principal component analysis therefore indicate that Gronwall and Wrightson's (1981) claim that PASAT performance is independent of general intelligence is not tenable. This conclusion is strongly supported by the results obtained from the confirmatory factor analyses in which competing models of the relationship between PASAT and components of WAIS-R performance were compared statistically. The initial model expressed the present authors' hypothesis that PASAT indexes both general intelligence and attention/concentration ability factors. The initial model had good fit and supported the results obtained by EFA. It can be seen from Fig. 1 that PASAT had a substantial loading on general intelligence and that, as in the EFA, this loading exceeded many of the WAIS-R subtests. It will be noted that PASAT's loadings on both the general factor and attention/concentration are lower in this CFA model than were obtained by EFA. However, this is true for all manifest variables in the model and occurs because the flexibility of CFA permitted simultaneous identification of the general and specific factors.

Models expressing alternative hypotheses were also tested. The first of these (Model 2) expressed the hypothesis that PASAT is entirely unrelated to factors 
underlying WAIS-R performance. Model 3 expressed the hypothesis that PASAT is solely a measure of attention/concentration; therefore it can be viewed as operationalising Gronwall and Wrightson's (1981) claim that PASAT is unrelated to general intellectual ability. Conversely, Model 4 represented the hypothesis that PASAT is solely an indicator of general intelligence. This model therefore was at the opposite extreme to Model 3 and expressed the hypothesis that all shared variance in PASAT was $g$ variance; i.e. it contained no specific attentional variance. All of these models had significantly poorer fit than the initial model so that a comparison between them is of limited value. However, in view of Gronwall and Wrightson's (1981) claim and the common assumption that PASAT predominantly measures attentional capacity, it is worth noting that the deterioration in fit was substantially greater (as indicated by the difference in Chi-square) when PASAT was constrained to load on the attention/concentration factor alone than when it was constrained to load on the general factor alone. It can also be seen from Figure 1 that PASAT's loading on $g(.664)$ exceeds its loading on attention/concentration (.440).

The present factor analytic results are consistent with Deary et al.'s. (1991) study of the relationship between WAIS-R performance and a two-trial version of PASAT, in which PASAT was found to load highly on general intelligence. Deary et al. used a short-form WAIS-R consisting of nine subtests and, as Digit Span was omitted, their results are not directly comparable with the present study. However, after rotation PASAT and Arithmetic did load on a third factor which Deary et al. identified as the attention/concentration factor.

In contrast to the convergence between the present findings and those of Deary et al., Roman et al.'s (1991) study of PASAT led these authors to conclude that, "for practical purposes IQ appears to bear a minimal relationship to PASAT performance" (p. 38). In this latter study the averaged correlation between WAIS-R and the four PASAT trials was a modest .30 (this averaged correlation was calculated by the present authors). The discrepancy between these results and those of the present study may be explained by differences in methodology. First, Roman et al. computed the correlation between an ageadjusted measure of intelligence and raw PASAT scores. As age exerts a significant effect on PASAT and WAIS-R performance, this procedure would attenuate the correlation between these latter variables. Therefore, we would suggest that these variables should be treated consistently; either scores on both measures should be age-corrected or both should not. In the present study the latter option was followed by using subtest scaled scores in the exploratory and confirmatory factor analyses.

The second important difference between these studies lies in the number of WAIS-R subtests administered. In the present study a full-length WAIS-R was completed by all participants whereas Roman et al. used a two-subtest shortform (Vocabulary and Block Design). The use of this short-form precluded moving beyond the manifest variables to examine the relationship between 
PASAT and the latent variables underlying WAIS-R performance. More significantly, the short-form assessed a limited range of intellectual ability. As a result the validity and reliability of the WAIS-R measure employed by Roman et al. would be lower than a full-length WAIS-R. Both these factors would serve to further attenuate the correlation with PASAT performance. Finally, Roman et al. computed the correlation between the WAIS-R and individual PASAT trials. The reliability of scores on an individual trial will be substantially less than for total PASAT scores. As a number of aspects of Roman et al.'s procedures conspired to attenuate the strength of the relationship, their results are not as seriously at odds with those of the present study and Deary et al. (1991) as they may first appear.

One of the subsidiary aims of the present study was to examine the relationship between age and PASAT performance. Age had a modest linear relationship with PASAT scores such that lower scores were associated with increasing age. This is consistent with previous studies of PASAT (e.g. Brittain et al., 1991) and other measures of attention and speed of information processing (Salthouse, 1991). It is however, at odds with Ward's (1997) report of a PASAT advantage in an elderly sample when compared with young adults. This latter study raised the possibility that there would be a non-linear relationship such that elderly and young adults would perform more poorly than middle-aged participants (in the latter case because of poor knowledge of number bonds). Such a possibility and other potential forms of non-linear relationship were allowed for in the present study by adding polynomial functions of age to the regression model. This revealed that any non-linear trends in the data failed to achieve statistical significance. However, the present results do not rule out the possibility that cohort differences in knowledge of number bonds serve to attenuate the relationship between age and attention/speed of information processing when PASAT is used as a measure of these constructs; it is worth noting that the strength of the PASAT/age relationship in the present study is modest when compared with that found for alternative measures of these constructs in which knowledge of number bonds plays no part (Salthouse, 1991).

A further subsidiary aim was to present additional normative data for the PASAT. The present sample size was relatively modest, however the sample was reasonably representative of the general adult population in the UK in terms of social class and sex ratio and was larger than the original normative sample. Although these norms and the original norms have some utility, the present factor analytic results also suggest that conventional normative data should be supplemented by attempts to obtain individualised comparison standards when interpreting PASAT performance in clinical practice. To assist in this process regression equations were built which allow clinicians to compare PASAT performance with a client's estimated premorbid performance and current general level of intellectual functioning. 
The use of these regression equations can be illustrated by the example of a client (RB) drawn from one of the present authors' case files. RB is a 34-yearold male with a university level education, previously employed as a senior marketing executive. He suffered a severe closed head injury as a result of a road traffic accident. Length of post-traumatic amnesia was 21 days. At 8 months post-injury RB's NART error score was 8 and WAIS-R FSIQ was 98; his score on Gronwall and Wrightson's original version of PASAT was 102. Entering RB's age and NART error score into equation 1 in Table 6 yielded an estimated premorbid PASAT score of 175; thus the discrepancy between predicted and obtained scores was 73 . This suggests an acquired deficit in attention/concentration as it can be seen from Table 7 that less than $5 \%$ of the healthy sample would be expected to exhibit a discrepancy of this magnitude (i.e. a discrepancy of 57.2 is sufficient to exceed $95 \%$ of the healthy sample).

It is notable that RB's performance does not appear so extreme when compared against the conventional normative data in Table 2, a PASAT score of 84 would be required to fall below $95 \%$ of the 30-49 year-old's normative group (i.e. a score which was $1.64 \mathrm{SD}$ units below the mean). This reinforces Lezak's (1995) emphasis on the usefulness of individual comparison standards when attempting to identify neuropsychological deficits. RB is an example of a client of above average premorbid ability whose current attentional capacity is below expectations. Conversely, given the strength of the relationship between PASAT and general ability, a low PASAT score does not necessarily represent an acquired deficit in a client who had only modest premorbid cognitive resources; i.e. a PASAT score which is significantly below the mean when a client is compared against a normative standard may not yield a large discrepancy when the individual comparison standard is employed.

Entry of RB's WAIS-R FSIQ and age into equation 2 in Table 6 produced an estimated PASAT score of 145; the difference between predicted and obtained PASAT scores was therefore 43 . Less than $10 \%$ of the healthy sample would be expected to show a discrepancy of this magnitude. Therefore, there is some evidence that the attention/concentration deficit qualified as a differential deficit, i.e. it was greater than his current averaged level of deficit on a standard measure of intellectual ability. There was also convergent evidence of attention/concentration difficulties from RB's WAIS-R subtest scores; Arithmetic and Digit Span, together with Digit Symbol, were the poorest performed subtests.

The method outlined for comparing a client's obtained score with that predicted by regression equations is widely used in clinical neuropsychology. However, the reader should be aware that the correct procedure is to form prediction limits based on what is termed the "standard error of a single $\hat{Y}$ (see Cohen \& Cohen, 1983). The difference between the two methods is negligible when scores on the predictors are relatively close to their respective means, but will diverge when scores on the predictors are extreme (the prediction limits 
become wider at the extremes because of the error in estimating the population regression coefficients from the sample regression coefficients). The difficulty in using prediction limits is that, when there is more than one predictor (as in the present case), the calculations required are formidable; e.g. they involve the inverse of the correlation matrix of predictor variables. Therefore, we have written a computer program for PCs which performs the necessary calculations for the two regression equations featured in this paper. A compiled version of this program can be downloaded from the first author's website at the following address: http://www.psyc/abdn.ac.uk/homedir/jcrawford/jcrawford.htm.

The majority of research on the NART and its variants has focused on its use as a means of estimating premorbid intelligence and has thus employed the WAIS or WAIS-R as the criterion variable (see Crawford, 1992; O'Carroll, 1995). The present study represents one of the few attempts to employ the NART as a means of estimating premorbid performance for tests designed more specifically for use in neuropsychological assessment. A NART equation is available for estimating premorbid performance on the FAS verbal fluency task (Crawford, Moore \& Cameron, 1992) but there are many other tests routinely used in the assessment of $\mathrm{CHI}$ clients for which the utility of this approach could be examined.

Finally, the PASAT has proved itself to be a useful clinical instrument in work with head-injured populations. For example, McMillan and Glucksman (1987) compared a mild head-injured sample with healthy controls on a battery of neuropsychological tests and reported that PASAT was the only measure which yielded a statistically significant difference. Furthermore, Brooks et al. (1987) found that PASAT was a powerful predictor of later employment status following a closed head injury. We do not view the results of the present study as seriously undermining the utility of the test. However, it would appear that, despite claims to the contrary, PASAT performance is not independent of general intellectual ability; this knowledge should guide clinical interpretation of the test. We are therefore in agreement with Brittain et al. (1991) who advised that, "... the age and intellectual functioning of subjects must be considered when interpreting PASAT scores" (p. 163). The regression equations provided in the present study should assist in this process as they allow clients' PASAT scores to be compared quantitatively with their current general intellectual ability and estimated premorbid intellectual ability, whilst simultaneously factoring in age.

\section{REFERENCES}

Anastasi, A. (1990). Psychological Testing (6th ed.). New York: Macmillan.

Atkinson, L., Cyr, J.J., Doxey, N.C.S., \& Vigna, C.M. (1989). Generalizability of WAIS-Rfactor structure within and between populations. Journal of Clinical Psychology, 45, 124-129. 
Bentler, P. (1989). EQS structural equations program manual. Los Angeles, CA: BMPD Statistical Software.

Bentler, P.M., \& Wu, E.J.C. (1993). EQS/Windows user's guide, Version 4. Cork: BMPD Statistical Software.

Brittain, J.L., La Marche, J.A., Reeder, K.P., Roth, D.L., \& Boll, T.J. (1991). Effects of age and IQ on paced auditory serial addition task (PASAT) performance. The Clinical Neuropsychologist, 5, 163-175.

Brooks, D.N., McKinlay, W., Symington, C., Beattie, A., \& Campsie, L. (1987). Return to work within the first seven years of severe head injury. Brain Injury, 1, 5-15.

Cohen, J., \& Cohen, P. (1983). Applied multiple regression/correlation analysis for the behavioural sciences (2nd ed.). Hillsdale NJ: Lawrence Erlbaum Associates Inc.

Crawford, J.R. (1992). Current and premorbid intelligence measures in neuropsychological assessment. In J.R. Crawford, W. McKinlay, \& D.M. Parker (Eds.), A handbook of neuropsychological assessment, (pp. 21-49). Hove, UK: Lawrence Erlbaum Associates Ltd.

Crawford, J.R., Allan, K.M., Stephen, D.W., Parker, D.M., \& Besson, J.A.O. (1989). The Wechsler Adult Intelligence Scale-Revised (WAIS-R): factor structure in a UK sample. Personality and Individual Differences, 10, 1209-1212.

Crawford, J.R., Besson, J.A.O., \& Parker, D.M. (1988). Estimation of premorbid intelligence in organic conditions. British Journal of Psychiatry, 153, 178-181.

Crawford, J.R., Moore, J.W., \& Cameron, I.M. (1992). Verbal fluency: a NART-based equation for the estimation of premorbid performance. British Journal of Clinical Psychology, 31, 327-329.

Deary, I.J., Langan, S.J., Hepburn, D.A., \& Frier, B.M. (1991). Which abilities does the PASAT test? Personality and Individual Differences, 12, 983-987.

Egan, V. (1988). PASAT: observed correlations with IQ. Personality and Individual Differences, 1, 179-180.

Gronwall, D. (1977). Paced auditory serial-addition task: a measure of recovery from concussion. Perceptual and Motor Skills, 44, 367-373.

Gronwall, D.M., \& Sampson, H. (1974). The psychological effects of concussion. Auckland: University Press.

Gronwall, D.M., \& Wrightson, P. (1974, September 14). Delayed recovery of intellectual function after minor head injury. Lancet, 605-609.

Gronwall, D.M.A., \& Wrightson, P. (1981). Memory and information processing capacity after closed head injury. Journal of Neurology, Neurosurgery, and Psychiatry, 44, 889-895.

Gustafsson, J., \& Balke, G. (1993). General and specific abilities as predictors of school achievement. Multivariate Behavioural Research, 28, 407-434.

Leckliter, I.N., Matarazzo, J.D., \& Silverstein, A.B. (1986). A literature review of factor analytic studies of the WAIS-R. Journal of Clinical Psychology, 42, 332-342.

Levin, H.S., Benton, A.L., \& Grossman, R.G. (1982). Neurobehavioural consequences of closed head injury. New York: Oxford University Press.

Levin, H.S., Mattis, S., Ruff, R.M., Eisenberg, H.M., Marshall, L.F., Tabaddor, K., High, W.M., \& Frankowski, R.F. (1987). Neurobehavioural outcome following minor head injury. Journal of Neurosurgery, 66, 234-243.

Lezak, M.D. (1995). Neuropsychological assessment (3rd Ed). New York: Oxford University Press.

Loehlin, J.C. (1987). Latent variable models. An introduction to factor, path, and structural analysis. Hillsdale NJ: Lawrence Erlbaum Associates Inc.

McMillan, T.M., \& Glucksman, E.E. (1987). The neuropsychology of moderate head injury. Journal of Neurology, Neurosurgery and Psychiatry, 50, 394-397.

McKinlay, W.W., \& Gray, J.M. (1992). Assessment of the severely head-injured. In J.R. Crawford, W. McKinlay, \& D.M. Parker (Eds.), A handbook of neuropsychological assessment, (pp. 363-378). Hove, UK: Lawrence Erlbaum Associates Ltd. 
Nelson, H.E. (1982). National Adult Reading Test (NART): Test Manual. Windsor: NFER Nelson.

Nelson, H.E., \& Willison, J. (1991). National Adult Reading Test manual (2nd ed.). Windsor, UK: NFER-Nelson.

O'Carroll, R.E. (1995). The assessment of premorbid ability. Neurocase, 1, 83-89.

O'Grady, K.E. (1983). A confirmatory maximum likelihood factor analysis of the WAIS-R. Journal of Consulting and Clinical Psychology, 51, 826-831.

Office of population, Censuses and Surveys (1980). Classification of occupations. London: HMSO.

Roman, D., Edwell, G.E., Buchanan, R.J., \& Patton, J.H. (1991). Extended norms for the Paced Auditory Serial Addition Task. The Clinical Neuropsychologist, 5, 33-40.

Salthouse, T.A. (1991). Theoretical perspectives on cognitive aging. Hillsdale NJ: Lawrence Erlbaum Associates Inc.

van Zomeren, A.H., \& Brouwer, W.H. (1992). The assessment of attention. In J.R. Crawford, D.M. Parker, \& W.W. McKinlay (Eds.), A handbook of neuropsychological assessment, (pp. 241-258). Hove, UK: Lawrence Erlbaum Associates Ltd.

Ward, T. (1997). A note of caution for clinicians using the Paced Auditory Serial Addition Task. British Journal of Clinical Psychology, 36, 303-307.

Wechsler, D. (1981). Manual for the Wechsler Adult Intelligence Scale-Revised. New York: Psychological Corporation. 